\title{
Special Issue: Green Networking and Computing, Part 1 of 2
}

The Internet was born as a simple, small-scale, communication system for transmitting low bit-rate traffic between machines. Today it is a large, dynamic, heterogeneous collection of interconnected systems that can be used for many different types of communications among any interested party connected to it. The term Internet includes both the "core Internet" (i.e., Internet Service Provider networks) and the "edge Internet" (i.e., corporate, data centres, private and community networks). Clearly, such a ubiquitous network paradigm comes at a no negligible cost.

It has been estimated that the global Information and Communications Technology (ICT) energy consumption amounts to $7 \%$ of the entire electricity production, while the energy requirements of data centers and network equipment are foreseen to grow with a yearly rate of $12 \%$. Furthermore, with an ever-increasing demand for bandwidth, connection quality and end-to-end interactivity, computer networks and mobile devices are in need of more and more sophisticated and power-hungry technologies.

The reduction of the energy consumption and the environmental impact of Green House Gas (GHG) emissions is nowadays a matter of great interest in the worldwide conscience, and among the most important challenges facing our civilization. Local area networks, broadband wireless technologies, long-haul systems and other networks cannot be designed, produced and commercialised without highlighting their advancement in the energy usage.

This Special Issue on "Green networking and computing" brings together state-of-the-art research contributions that address many aspects of the enabling technologies for greening the Internet. The nine selected papers cover various sub-fields: (i) energy-oriented protocols and technologies for broadband access networks, (ii) traditional IP over WDM core networks with energy considerations, and (iii) novel technologies with energy as a new constraint for core networks.

The first three papers deal with energy efficiency in the access segment of the network. In the first paper about Energy efficient multicasting in TDMA PONs, L. Valcarenghi, I. Cerutti and P. Castoldi propose an approach where energy is saved by setting to sleep mode the ONUs that are not subscribed to a multicast group, i.e., not receiving data. Based on this intuition the authors propose a multicast group scheduling approach that minimizes the number of transitions from sleep to active mode. Their results show that with multicast transmissions it is possible to achieve better energy consumption figures than with current, broadcast-based, PON access solutions. Next, in the paper on Energy consumption of subscriber devices in broadband network, K. Borzycki provides estimates on the impact of the deployment of fast fixed broadband networks on the energy consumption of subscriber's electronic devices. The author concludes that the progress in cutting device power consumption and the use of energy-efficient portable devices shall considerably mitigate any negative effects of the increasing interest in fast broadband and video services. The last article of this first part is entitled "Energy Efficient Ethernet" enhanced with a QoS-aware sleep mode controller by P. Testa, A. Germoni and M. Listanti. The authors propose a method to shape the traffic transmission at switches output ports in order to reduce the power consumption of the network interface by exploiting and improving the Energy Efficient Ethernet (EEE) low power idle standard (IEEE802.3az).

The next three papers published in Part 2 of the special issue (Journal of High Speed Networks, Volume 19, Number 1, 2013), propose energy-aware strategies for traditional optical core networks. In A distributed framework for energy-efficient lightpaths in computational grids, D. Tafani, B. Kantarci, H.T. Mouftah, C. McArdle and 
L.P. Barry address the problem of energy-efficient lightpath establishment in computational grid networks. The proposed solution is based on an adaptive and distributed scheme in which the reduction of energy consumption is achieved by means of an Energy Saving Mode implemented at network nodes. Next, in the paper on Energyefficiency of protected IP-over-WDM networks with sleep-mode devices by F. Musumeci, M. Tornatore, J.L. Vizcaino, Y. Ye and A. Pattavina, the authors address the problem of power efficiency of protected IP-over-WDM networks. Their study provides a comprehensive comparison of four different protection strategies, i.e., sharedlink, shared-path, dedicated-link and dedicated-path protection. The considered scenario allows for resources that are not used, or reserved for protection purpose only, to be put in sleep-mode. The authors show that, with their proposed approach, significant power savings can be obtained for all the protection strategies analysed. Caching in green IP over WDM networks by N. Osman, T. Elgorashi and J. Elmirghani concludes this second set of articles. The authors investigate the minimization of the power consumption obtained by caching content in IP over WDM networks. In particular, the authors formulate a MILP model and propose a genetic-based algorithm to optimize the cache size of the network nodes at different times of the day. Results reveal that the proposed method obtains considerable gain in power savings.

The final three papers are related to new technologies in core networks. In the paper on Digital subcarrier optical networks and cross-connects by R. Hui, W. Huang, Y. Zhang, M. Hameed, M. Razo, M. Tacca and A. Fumagalli, the authors describe a rate flexible network transport solution for sub-wavelength circuit provisioning between edge node pairs. Sub-wavelength circuits are obtained by concatenating spectrally efficient digital subcarrier channels along the network path, with each subcarrier frequency carrying only a fraction of the wavelength bandwidth. The authors show that, compared to OTN/SONET/SDH transport network solutions, their solution offers the possibility to have tangible cost and electric power consumption reductions. Then, in Scalable and energy-efficient packet switches based on multi-granular forwarding operations, P. Testa, A. Germoni and M. Listanti focus on designing new packet switch architectures able to meet the requirements of low power consumption, high scalability, and simplified operations. The authors propose a solution based on Ethernet aggregation and packet switch forwarding mechanism, which allows the reduction of energy consumption by means of reducing the processing of transit traffic. Finally, the paper on Adaptive hybrid optical switching: Performance and energy efficiency by S. Aleksic, M. Fiorani and M. Casoni presents a Hybrid Optical Switching (HOS) network concept and investigates several architectures and realization options for AHOS nodes. According to the authors, the proposed framework results in an improvement of both energy and data transport efficiency because of its ability to always chose (i) the most appropriate switching method, and (ii) the less power consuming elements used for transmission, while temporarily inactive elements are put in a low-power (i.e., sleep) mode.

\author{
The Guest Editors \\ Davide Careglio \\ Universitat Politècnica de Catalunya (UPC), \\ Barcelona, Spain \\ E-mail: careglio@ac.upc.edu (Corresponding author) \\ Paolo Monti \\ Royal Institute of Technology (KTH), Kista, Sweden \\ E-mail:pmonti@kth.se
}

Mirosław Klinkowski

National Institute of Telecommunications (NIT), Warsaw, Poland

E-mail: mklinkow@itl.waw.pl 